\title{
Antimicrobial Resistance Trends among Community-Acquired Respiratory Tract Pathogens in Greece, 2009-2012
}

\author{
Sofia Maraki and Ioannis S. Papadakis \\ Department of Clinical Microbiology, Parasitology, Zoonoses and Geographical Medicine, University Hospital of Heraklion, \\ 71110 Heraklion, Crete, Greece \\ Correspondence should be addressed to Sofia Maraki; sofiamaraki@in.gr
}

Received 23 August 2013; Accepted 26 November 2013; Published 27 January 2014

Academic Editors: D. P. Levine and G. A. Rocha

Copyright (C) 2014 S. Maraki and I. S. Papadakis. This is an open access article distributed under the Creative Commons Attribution License, which permits unrestricted use, distribution, and reproduction in any medium, provided the original work is properly cited.

The aim of the present study was to determine the antimicrobial resistance trends of respiratory tract pathogens isolated from patients with community-acquired respiratory tract infections (CARTIs) in Crete, Greece, over a 4-year period (2009-2012). A total of 588 community-acquired respiratory pathogens were isolated during the study period. Streptococcus pneumoniae was the most common organism responsible for $44.4 \%$ of CARTIs, followed by Haemophilus influenzae (44.2\%) and Moraxella catarrhalis (11.4\%). Among S. pneumoniae, the prevalence of isolates with intermediate- and high-level resistance to penicillin was $27.2 \%$ and $12.3 \%$, respectively. Macrolide resistance slightly decreased from $29.4 \%$ over the period $2009-2010$ to $28.8 \%$ over the period $2011-$ 2012. Multiresistance was observed among 56 (54.4\%) penicillin nonsusceptible isolates. A nonsignificant increase in resistance of $H$. influenzae isolates was noted for $\beta$-lactams, cotrimoxazole, and tetracycline. Among the $67 \mathrm{M}$. catarrhalis tested, 32 produced beta-lactamase and were resistant to ampicillin. Macrolide resistance decreased over the study period. All isolates were susceptible to amoxicillin + clavulanic acid, chloramphenicol, rifampicin, and the fluoroquinolones. Although a decreasing trend in the prevalence of resistance of the three most common pathogens involved in CARTIs was noted, continuous surveillance of antimicrobial susceptibility at the local and national level remains important, in order to guide appropriate empirical antimicrobial therapy.

\section{Introduction}

Community-acquired respiratory tract infections (CARTIs) such as acute otitis media, acute sinusitis, acute exacerbations of chronic bronchitis, and community-acquired pneumonia are very common causes of morbidity and are among the leading reasons for physicians' office visits worldwide [1]. Due to the severity of these infections and the difficulties in determining the bacterial etiology and the antimicrobial susceptibility, treatment is often empirical, consisting usually of orally administered agents. The choice of empiric antimicrobial chemotherapy is guided by the clinical presentation, the severity of the infection, and epidemiologic data, comprising the causative organisms and their susceptibility to antimicrobial agents $[2,3]$.

Streptococcus pneumoniae, Haemophilus influenzae, and Moraxella catarrhalis are the major pathogens implicated in community-acquired respiratory tract infections. The increasing prevalence of antimicrobial resistance among these species remains a global problem complicating the management of CARTIs $[4,5]$.

Of particular concern are the emergence and dissemination of $S$. pneumoniae strains resistant to penicillin and multidrug resistant (MDR) to several antibiotic classes [6]. In $H$. influenzae and M. catarrhalis $\beta$-lactamase (BL) production has been the primary mechanism for bacterial resistance to beta-lactams $[7,8]$. However, rare $\beta$-lactamase negative ampicillin-resistant (BLNAR) H. influenzae isolates have been reported [7].

In the present study, we describe the antimicrobial susceptibility patterns of community-acquired respiratory tract pathogens that were isolated in the microbiology laboratory the last 4 years.

\section{Materials and Methods}

We prospectively tested in vitro all isolates of $H$. influenzae, S. pneumoniae, and M. catarrhalis that were recovered from 
TABLE 1: Distribution of community-acquired respiratory pathogens by study year (2009-2012).

\begin{tabular}{lccccc}
\hline & 2009 & 2010 & 2011 & 2012 & $2009-2012$ \\
\hline S. pneumoniae & $73(42.5 \%)$ & $63(49.6 \%)$ & $62(44 \%)$ & $63(42.6 \%)$ & $261(44.4 \%)$ \\
H. influenzae & $79(45.9 \%)$ & $51(40.2 \%)$ & $61(43.2 \%)$ & $69(46.6 \%)$ & $260(44.2 \%)$ \\
M. catarrhalis & $20(11.6 \%)$ & $13(10.2 \%)$ & $18(12.8 \%)$ & $16(10.8 \%)$ & $67(11.4 \%)$ \\
\hline Total & 172 & 127 & 141 & 148 & 588 \\
\hline
\end{tabular}

patients with CARTIs at the University Hospital of Heraklion over the period 1/2009-12/2012. One bacterial isolate was tested per patient. Isolates collected either from nonhospitalized patients or from hospitalized patients within 48 hours of admission were included. Organisms were recovered from patients with sinusitis, otitis media, community-acquired pneumonia, acute bacterial exacerbation of chronic bronchitis, and acute exacerbation of chronic obstructive airways disease. Blood, pleural fluid, bronchoalveolar fluid, sputum, middle ear fluid, and sinus aspirate cultures were considered acceptable sources for study isolates.

2.1. Identification and Antimicrobial Susceptibility Testing. S. pneumoniae was identified on the basis of colony and microscopic morphology, hemolytic reactions on sheep blood agar medium, catalase test, optochin susceptibility, bile solubility, and biochemical profile using the Vitek 2 automated system (BioMérieux). H. influenzae was identified on the basis of hemolytic reactions on horse blood agar, growth requirement for $\mathrm{X}$ and $\mathrm{V}$ factors, and by the use of Vitek 2 automated system (BioMérieux, Marcy l' Etoile, France). Identification of $M$. catarrhalis was based on colony and microscopic morphology, oxidase test, and by the use of Vitek 2 automated system (BioMérieux). For $S$. pneumoniae isolates, minimum inhibitory concentrations (MICs) were determined using the E-test method, for penicillin, cefuroxime, cefotaxime, ceftriaxone, cefepime, imipenem, meropenem, erythromycin, clarithromycin, roxithromycin, azithromycin, clindamycin, ciprofloxacin, levofloxacin, moxifloxacin, chloramphenicol, tetracycline, cotrimoxazole (SXT), and vancomycin. Results were interpreted according to the 2012 Clinical and Laboratory Standards Institute criteria (CLSI) [9]. The double-disk diffusion method with erythromycin $(15 \mu \mathrm{g})$ and clindamycin $(2 \mu \mathrm{g})$ (Bio-Rad, Marnes-la-Coquette, France) and MIC data were used for the determination of macrolide resistance phenotype [9]. Resistance to both erythromycin and clindamycin is characteristic of a $\mathrm{CMLS}_{\mathrm{B}}$ phenotype, while blunting of the clindamycin inhibition zone near the erythromycin disc is indicative of an $\mathrm{iMLS}_{\mathrm{B}}$ phenotype. Resistance to erythromycin and susceptibility to clindamycin with no blunting are characteristic of the $\mathrm{M}$ phenotype. Multiresistance was defined as resistance to three or more classes of antibiotics.

For $H$. influenzae and $M$. catarrhalis antimicrobial susceptibility tests were performed by employing the disk diffusion method and the results were interpreted according to the 2012 CLSI criteria $[9,10]$. Intermediate isolates were grouped along with resistant isolates. The antibiotics that were tested against $H$. influenzae isolates are the following: ampicillin, amoxicillin + clavulanic acid, clarithromycin, chloramphenicol, tetracycline, SXT, ciprofloxacin, ofloxacin, and moxifloxacin. The antibiotics that were tested against $M$. catarrhalis isolates are the following: amoxicillin, amoxicillin + clavulanic acid, chloramphenicol, tetracycline, clarithromycin, SXT, rifampicin, ciprofloxacin, and moxifloxacin. Beta-lactamase (BL) production was assayed bythe nitrocefin test (Oxoid, Basingstoke, UK). BLNAR H. influenzae strains were defined as BL-negative strains that were resistant to ampicillin (MIC $\geq 4 \mu \mathrm{g} / \mathrm{mL}$ ) [9].

In order to test for differences in the antibiotic susceptibility patterns between the earlier and later study years for a given antibiotic, we compared the resistance profiles of respiratory isolates of the period 2009-2010 with those of the period 2011-2012.

2.2. Statistical Analysis. Statistical analysis was conducted by Fisher's exact and Kruskal-Wallis tests, as appropriate. Statistical significance was set at $P<0.05$. All analyses were performed with Graphpad Prism, V.4 (GraphPad Software Inc., CA, USA).

\section{Results}

3.1. Bacterial Isolates and Patients' Demographics. A total of 261 S. pneumoniae, $260 \mathrm{H}$. influenzae, and $67 \mathrm{M}$. catarrhalis were isolated during the period 1/2009-12/2012 from patients with CARTIs. The distribution of respiratory pathogens by study year is shown in Table 1. Sixty-three percent of all isolates were obtained from male patients (Table 2). Most of the $S$. pneumoniae strains were most frequently isolated from pediatric patients $\leq 5$ years of age, while $H$. influenzae and $M$. catarrhalis were derived from adults $\geq 61$ years of age (Table 2).

3.2. Antibiotic Resistance among S. pneumoniae Isolates. In vitro susceptibility data for 19 antimicrobial agents are presented in Table 3 . The proportion of penicillin nonsusceptible (PNSP) isolates was 39.5\% (27.2\% with intermediate resistance and $12.3 \%$ with high-level resistance). Isolates with intermediate and high-level resistance became more frequent over time, from $24.3 \%$ over the period 2009 to 2010 , increased to $30.4 \%$ over the years 2011 to 2012 , while isolates with high-level resistance decreased the second half of the study period $(P=0.09)$ (Table 3$)$. Resistance to the third generation cephalosporins decreased over the two two-year time periods, from $6.6 \%$ to $1.6 \%$ for cefotaxime and from $5.9 \%$ to $0.8 \%$ for ceftriaxone. Similarly, there was a trend for nonsignificant decrease in cefepime resistance 
TABle 2: Numbers of isolates of $H$. influenzae, S. pneumoniae, and M. catarrhalis, grouped according to gender and age.

\begin{tabular}{lccc}
\hline & $\begin{array}{c}\text { S. pneumoniae } \\
(n=261)\end{array}$ & $\begin{array}{c}\text { H. influenzae } \\
(n=260)\end{array}$ & $\begin{array}{c}\text { M. catarrhalis } \\
(n=67)\end{array}$ \\
\hline Gender & & & \\
$\quad$ Male & 166 & 165 & 43 \\
$\quad$ Female & 95 & 95 & 24 \\
Age groups & & & \\
(years) & & & \\
$0-5$ & 110 & 49 & 12 \\
$6-15$ & 24 & 46 & 11 \\
$16-45$ & 24 & 47 & 9 \\
$46-60$ & 26 & 25 & 5 \\
$\geq 61$ & 77 & 93 & 30 \\
\hline
\end{tabular}

(including intermediately and fully resistant strains) that decreased from $18.4 \%$ to $16 \%(P=0.15)$. Resistance to erythromycin was detected in 76 isolates (29.1\%). Among them, $\mathrm{M}$ and $\mathrm{CMLS}_{\mathrm{B}}$ phenotypes were observed in 40 (52.6\%) and $22(28.9 \%)$ strains, respectively, by the use of the double disk approximation test. Additionally, 14 strains (18.4\%) with the iMLS ${ }_{\mathrm{B}}$ phenotype were detected. Erythromycin resistance decreased from $29.4 \%$ over the years 2009 to 2010 to $28.8 \%$ over the period 2011 to 2012, but this was not significant $(P=$ 1.00) (Table 3). Only two isolates, one in each study period, were resistant to newer fluoroquinolones, while all isolates were susceptible to vancomycin (Table 3 ).

Multiresistance was observed among 56 (54.4\%) PNSP strains. The predominant phenotype of multidrug resistance was nonsusceptible to penicillin, erythromycin, clindamycin, and tetracycline $(28.6 \%)$, and the second most frequent phenotype was nonsusceptible to penicillin, erythromycin, tetracycline, and SXT (26.8\%) (Table 4).

3.3. Antibiotic Resistance among H. influenzae Isolates. Of the $260 \mathrm{H}$. influenzae isolates, 36 (13.8\%) produced $\beta$-lactamase. Ampicillin-resistant isolates represented $14 \%$, while amoxicillin + clavulanic acid-resistant isolates were only $0.8 \%$. Overall, 1 BLNAR strain was isolated. A nonsignificant increase in resistance of $H$. influenzae isolates was noted between the two study periods for ampicillin and amoxicillin + clavulanic acid. The same was true for clarithromycin, SXT, and tetracycline (Table 5).

3.4. Antibiotic Resistance among M. catarrhalis Isolates. Among the 67 isolates tested, almost half of them (32 isolates) were resistant to ampicillin and produced beta-lactamase. Macrolide resistance decreased from $36.4 \%$ in $2009-2010$ to $29.4 \%$ in 2011-2012. The reverse trend was noted for cotrimoxazole. Only one isolate was resistant to tetracycline and all 67 isolates were susceptible to amoxicillin + clavulanic acid, chloramphenicol, rifampicin, and the two fluoroquinolones tested (Table 5).

\section{Discussion}

The results of the present study provide an update on resistance trends amongst pathogens that cause CARTIs in our area. Increasingly, resistance of $S$. pneumoniae to penicillin is of special concern, because this agent has been considered the therapy of choice for pneumococcal CARTIs, due to its efficacy and low cost $[4,6]$. Although resistant strains of $S$. pneumoniae are detected universally, the incidence of resistance varies markedly between countries and regions. Data from the Alexander project reported penicillin resistance levels in S. pneumoniae ranging from 7.8\% in Eastern Europe through $25 \%$ in the USA to $38.8 \%$ in several parts of Asia in 1998-2000 [4].

The first study conducted in Greece in the early 1990, evaluating the antimicrobial susceptibilities of 1,002 clinical isolates of $S$. pneumoniae deriving from patients with community-acquired pneumonia, reported resistance rates of $14 \%$ for penicillin [11]. A recent study from the same centre showed an increase of $4.5 \%$ in penicillin resistance [12]. In the present study, the percentage of intermediately resistant and resistant isolates decreased from $41.9 \%$ to $36.8 \%$ over the two study periods. More specifically, the percentage of highly resistant isolates dropped from $17.6 \%$ to $6.4 \%$. Additionally, the low rates of resistance to third-generation cephalosporins favour their use as the first-choice empirical treatment for community-acquired pneumonia necessitating hospital admission [13]. In the present study 54.4\% of PNSP strains were found to be multidrug resistant, a rate similar to that previously reported [14]. Riedel et al. in their study in 15 European countries reported overall MDR to $15.8 \%$, varying from $0 \%$ in Denmark to $42.9 \%$ in Greece [15].

Resistance to macrolides, which reached $29.1 \%$ among pneumococcal isolates, renders empirical monotherapy with a macrolide not appropriate for treatment of patients with community-acquired pneumonia. The US (IDSA/ATS) guidelines recommend that in regions where macrolideresistance exceeds $25 \%$, macrolide monotherapy is not appropriate even for patients without comorbidities [16]. The M phenotype encoded by the mef gene predominated in Crete, a feature also prevailing in other areas in Greece, in the United States, and Canada but much rarer in other European countries, where the $M_{\mathrm{B}}$ phenotype, characterized by resistance to macrolides, lincosamides, and streptogramin, is most commonly encountered [17-19]. The high frequency of resistance to macrolides is due to their increased outpatient use for CARTIs. The relationship between macrolide resistance in S. pneumoniae and macrolide consumption has been shown by several investigators, reporting low resistance rates in low consuming areas as Scandinavia and high in Mediterranean countries with increased macrolide use, such as France, Spain, and Italy, where the respective prevalence are $58 \%, 29-35 \%$, and $32 \%-43 \%$ [15, 20-22].

Overall, 52 isolates $(19.9 \%)$ were resistant to cotrimoxazole, which is lower than that found in our previous study of the period 2001-2008 [14]. This finding is likely due to the decreased outpatient consumption of the drug in our area, 
TABle 3: Comparison of antibiotic resistance rates in S. pneumonia isolates over the periods 2009-2010 and 2011-2012.

\begin{tabular}{|c|c|c|c|c|c|c|c|c|c|c|c|c|c|}
\hline \multirow[b]{2}{*}{ Antibiotic } & \multicolumn{7}{|c|}{$2009-2010$} & \multicolumn{6}{|c|}{ 2011-2012 } \\
\hline & $\mathrm{MIC}_{50}$ & $\mathrm{MIC}_{90}$ & Range & $S \%$ & $I \%$ & $R \%$ & $\mathrm{MIC}_{50}$ & $\mathrm{MIC}_{90}$ & Range & $S \%$ & $I \%$ & $R \%$ & $P$ value \\
\hline Penicillin & 0.023 & 2 & $<0.016-4$ & 58.1 & 24.3 & 17.6 & 0.023 & 0.75 & $<0.016-4$ & 63.2 & 30.4 & 6.4 & 0.09 \\
\hline Cefuroxime & 0.023 & 3 & $<0.016-6$ & 72.1 & 1.4 & 26.5 & 0.023 & 2 & $<0.016-4$ & 80.8 & 2.4 & 16.8 & 0.10 \\
\hline Cefotaxime & 0.023 & 1 & $<0.016-2$ & 93.4 & 5.9 & 0.7 & 0.023 & 0.55 & $<0.016-1.5$ & 98.4 & 1.6 & - & 0.10 \\
\hline Ceftriaxone & 0.023 & 1 & $<0.016-1.5$ & 94.1 & 5.9 & - & 0.023 & 0.5 & $<0.016-1$ & 99.2 & 0.8 & - & 0.03 \\
\hline Cefepime & 0.064 & 2 & $0.0023-3$ & 81.6 & 14.7 & 3.7 & 0.125 & 2 & $0.016-6$ & 84 & 7.2 & 8.8 & 0.15 \\
\hline Imipenem & 0.016 & 0.19 & $0.003-0.75$ & 88.2 & 10.3 & 1.4 & 0.047 & 0.125 & $0.006-0.5$ & 94.4 & 5.6 & - & 0.10 \\
\hline Meropenem & 0.012 & 0.38 & $0.004-1$ & 88.2 & 8.8 & 2.9 & 0.032 & 0.125 & $0.006-1$ & 96 & 2.4 & 1.6 & 0.14 \\
\hline Erythromycin & 0.094 & $\geq 256$ & $0.047-\geq 256$ & 70.6 & - & 29.4 & 0.064 & $\geq 256$ & $0.016-\geq 256$ & 71.2 & - & 28.8 & 1.00 \\
\hline Clarithromycin & 0.094 & $\geq 256$ & $0.016-\geq 256$ & 70.6 & - & 29.4 & 0.047 & $\geq 256$ & $0.016-\geq 256$ & 71.2 & - & 28.8 & 1.00 \\
\hline Clindamycin & 0.125 & $\geq 256$ & $0.032-\geq 256$ & 89.7 & - & 10.3 & 0.064 & $\geq 256$ & $0.023-\geq 256$ & 82.4 & 1.6 & 16 & 0.10 \\
\hline Roxithromycin & 0.125 & $\geq 256$ & $0.047-\geq 256$ & 70.6 & - & 29.4 & 0.094 & $\geq 256$ & $0.016-\geq 256$ & 71.2 & - & 28.8 & 1.00 \\
\hline Azithromycin & 0.5 & $\geq 256$ & $0.125-\geq 256$ & 70.6 & - & 29.4 & 0.19 & $\geq 256$ & $0.023-\geq 256$ & 71.2 & - & 28.8 & 1.00 \\
\hline Ciprofloxacin & 0.75 & 1.5 & $0.385-\geq 32$ & 99.3 & - & 0.7 & 0.75 & 1 & $0.25-\geq 32$ & 99.2 & - & 0.8 & 1.00 \\
\hline Levofloxacin & 0.75 & 1 & $0.19-16$ & 99.3 & - & 0.7 & 0.75 & 1 & $0.19-\geq 32$ & 99.2 & - & 0.8 & 1.00 \\
\hline Moxifloxacin & 0.125 & 0.19 & $0.032-1.5$ & 99.3 & $0.7 \%$ & - & 0.125 & 0.19 & $0.047-3$ & 99.2 & - & 0.8 & 0.16 \\
\hline Chloramphenicol & 2 & 2 & $0.38-12$ & 99.3 & - & 0.7 & 2 & 3 & $0.75-32$ & 96.8 & - & 3.2 & 0.19 \\
\hline Tetracycline & 0.125 & 16 & $0.016-48$ & 74.3 & 1.4 & 24.3 & 0.25 & 32 & $0.064-64$ & 79.2 & - & 20.8 & 0.10 \\
\hline Cotrimoxazole & 0.19 & 1.5 & $0.016-\geq 32$ & 77.2 & 14.7 & 8.1 & 0.094 & 1.5 & $0.023-\geq 32$ & 83.2 & 9.6 & 7.2 & 0.10 \\
\hline Vancomycin & 0.38 & 0.5 & $0.25-0.5$ & 100 & - & - & 0.38 & 0.5 & $0.25-0.75$ & 100 & - & - & $\mathrm{NA}^{*}$ \\
\hline
\end{tabular}

${ }^{*}$ NA: not applicable.

TABLE 4: MDR phenotypes of the 56 pneumococcal isolates by study year.

\begin{tabular}{lcccc}
\hline MDR phenotype & 2009 & 2010 & 2011 & 2012 \\
\hline P, E, Te & 2 & 2 & 1 & 2 \\
P, E, SXT & 1 & 1 & 0 & 0 \\
P, E, CM, Te & 1 & 4 & 7 & 4 \\
P, E, CM, SXT & 0 & 0 & 0 & 1 \\
P, E, Te, SXT & 6 & 5 & 4 & 0 \\
P, E, CM, Te, SXT & 1 & 4 & 3 & 2 \\
P, E, CM, C, Te, SXT & 0 & 1 & 3 & 0 \\
P, C, Te, SXT & 0 & 0 & 0 & 1 \\
\hline Total & 11 & 17 & 18 & 10 \\
\hline
\end{tabular}

P: penicillin; E: erythromycin; Te: tetracycline; SXT: cotrimoxazole; $\mathrm{CM}$ : clindamycin; C: chloramphenicol.

since the connection between decreased consumption of SXT and decreased resistance in S. pneumoniae has been shown [23].

Fluoroquinolone resistance was rare among the S. pneumoniae isolates. In Greece, quinolone nonsusceptibility is low, although fluoroquinolones are widely used in the treatment of community-acquired infections [14, 17]. Conflicting reports exist regarding the association of fluoroquinolone use and their resistance. Some investigators reported low fluoroquinolone resistance rates despite high usage, while others found that increased usage of fluoroquinolones strictly correlates with increased incidence of resistance [24-27]. It has been also shown that the use of quinolones with limited antipneumococcal activity favours the emergence of resistant strains [28], while the more active fluoroquinolones, such as moxifloxacin and gatifloxacin, are potentially less likely to select for resistance mutations, if mutation prevention concentrations are considered in relation to achievable serum levels [29].

Regarding $H$. influenzae, production of $\beta$-lactamase in the current 4 -year study was found in $13.8 \%$ of the isolates, which is higher than the proportion shown previously [30] but similar to that reported in the Alexander Project [31]. Within the European countries, there is considerable variability in the prevalence of $\beta$-lactamase production by $H$. influenzae, with previous studies showing values from $<10 \%$ for Germany, Italy, The Netherlands, and Poland to $>30 \%$ for France, Portugal, and Romania [31, 32]. Interestingly, it has been suggested that $\beta$-lactamase prevalence in $H$. influenzae has decreased in recent years in some countries [7]. Only one BLNAR strain of $H$. influenzae was detected during the study period. Although the global prevalence of BLNAR H. influen$z a e$ strains is very low, their incidence is on rise [33, 34].

Almost one-third of the $H$. influenzae isolates were found resistant to macrolides, a finding consistent with that of other investigators, who also used pharmacokinetic/pharmacodynamic breakpoints to interprete susceptibility data for macrolides $[35,36]$. There was a noticeable decrease in the resistance of $H$. influenzae to SXT when the results of this study, where $72.7 \%$ of the isolates were susceptible to SXT, were compared with those from a previous study from the same area, where $62.3 \%$ of the isolates were susceptible to SXT [30]. This is likely due to the decreased use of SXT during the study period, although no significant connection between SXT use and resistance among $H$. influenzae 
TAble 5: Trends in antibiotic resistance of $H$. influenzae and $M$. catarrhalis isolates.

(a) Haemophilus influenza

\begin{tabular}{|c|c|c|c|c|c|c|c|}
\hline Antimicrobial agent & $\begin{array}{c}2009 \\
n=79 \\
\text { resistant }\end{array}$ & $\begin{array}{c}2010 \\
n=51 \\
\text { resistant }\end{array}$ & $\begin{array}{c}2011 \\
n=61 \\
\text { resistant }\end{array}$ & $\begin{array}{c}2012 \\
n=69 \\
\text { resistant }\end{array}$ & $\begin{array}{c}2009-10(\mathrm{~A}) \\
n=130 \\
\text { resistant }\end{array}$ & $\begin{array}{c}2011-12(\mathrm{~B}) \\
n=130 \\
\text { resistant }\end{array}$ & $\begin{array}{c}P \text { value } \\
\text { A versus B }\end{array}$ \\
\hline Ampicillin & $11(13.9 \%)$ & $5(9.8 \%)$ & $10(16.4 \%)$ & $10(14.5 \%)$ & $16(12.3 \%)$ & $20(15.4 \%)$ & 0.59 \\
\hline Amoxicillin + CA & $0(0 \%)$ & $1(2 \%)$ & $0(0 \%)$ & $1(0.7 \%)$ & $1(1.4 \%)$ & $1(1.4 \%)$ & 1.00 \\
\hline Clarithromycin & $25(31.6 \%)$ & $15(29.4 \%)$ & $17(27.9 \%)$ & $21(30.4 \%)$ & $40(30.8 \%)$ & $38(29.2 \%)$ & 0.89 \\
\hline Chloramphenicol & $0(0 \%)$ & $0(0 \%)$ & $0(0 \%)$ & $0(0 \%)$ & $0(0 \%)$ & $0(0 \%)$ & NA \\
\hline Tetracycline & $6(7.6 \%)$ & $4(7.8 \%)$ & $5(8.2 \%)$ & $6(8.7 \%)$ & $10(7.7 \%)$ & $11(8.5 \%)$ & 1.00 \\
\hline Cotrimoxazole & $18(22.8 \%)$ & $15(29.4 \%)$ & $19(31.1 \%)$ & $19(27.5 \%)$ & $33(25.4 \%)$ & $38(29.2 \%)$ & 0.57 \\
\hline Ciprofloxacin & $0(0 \%)$ & $0(0 \%)$ & $0(0 \%)$ & $0(0 \%)$ & $0(0 \%)$ & $0(0 \%)$ & NA \\
\hline Ofloxacin & $0(0 \%)$ & $0(0 \%)$ & $0(0 \%)$ & $0(0 \%)$ & $0(0 \%)$ & $0(0 \%)$ & NA \\
\hline Moxifloxacin & $0(0 \%)$ & $0(0 \%)$ & $0(0 \%)$ & $0(0 \%)$ & $0(0 \%)$ & $0(0 \%)$ & NA \\
\hline
\end{tabular}

CA: clavulanic acid; NA: not applicable.

(b) Moraxella catarrhalis

\begin{tabular}{|c|c|c|c|c|c|c|c|}
\hline Antimicrobial agent & $\begin{array}{c}2009 \\
n=20 \\
\text { resistant }\end{array}$ & $\begin{array}{c}2010 \\
n=13 \\
\text { resistant }\end{array}$ & $\begin{array}{c}2011 \\
n=18 \\
\text { resistant }\end{array}$ & $\begin{array}{c}2012 \\
n=16 \\
\text { resistant }\end{array}$ & $\begin{array}{c}2009-10(\mathrm{~A}) \\
n=33 \\
\text { resistant }\end{array}$ & $\begin{array}{c}2011-12(\mathrm{~B}) \\
n=34 \\
\text { resistant }\end{array}$ & $\begin{array}{c}P \text { value } \\
\text { A versus B }\end{array}$ \\
\hline Amoxicillin & $8(40 \%)$ & $8(61.5 \%)$ & $8(44.4 \%)$ & $8(50 \%)$ & $16(48.5 \%)$ & $16(47 \%)$ & 1.00 \\
\hline Amoxicillin + CA & $0(0 \%)$ & $0(0 \%)$ & $0(0 \%)$ & $3(18.7 \%)$ & $0(0 \%)$ & $0(0 \%)$ & NA \\
\hline Chloramphenicol & $0(0 \%)$ & $0(0 \%)$ & $0(0 \%)$ & $0(0 \%)$ & $0(0 \%)$ & $0(0 \%)$ & NA \\
\hline Tetracycline & $0(0 \%)$ & $0(0 \%)$ & $0(0 \%)$ & $1(6.3 \%)$ & $0(\%)$ & $1(2.9 \%)$ & 1.00 \\
\hline Clarithromycin & $7(35 \%)$ & $5(38.5 \%)$ & $4(22.2 \%)$ & $6(37.5 \%)$ & $12(36.4 \%)$ & $10(29.4 \%)$ & 0.60 \\
\hline Cotrimoxazole & $6(30 \%)$ & $2(15.4 \%)$ & $5(27.8 \%)$ & $6(37.5 \%)$ & $8(24.2 \%)$ & $11(32.3 \%)$ & 0.59 \\
\hline Rifampicin & $0(0 \%)$ & $0(0 \%)$ & $0(0 \%)$ & $0(0 \%)$ & $0(0 \%)$ & $0(0 \%)$ & NA \\
\hline Ciprofloxacin & $0(0 \%)$ & $0(0 \%)$ & $0(0 \%)$ & $0(0 \%)$ & $0(0 \%)$ & $0(0 \%)$ & NA \\
\hline Moxifloxacin & $0(0 \%)$ & $0(0 \%)$ & $0(0 \%)$ & $0(0 \%)$ & $0(0 \%)$ & $0(0 \%)$ & NA \\
\hline
\end{tabular}

CA: clavulanic acid; NA: not applicable.

isolates has been shown [23]. Tetracycline resistance was low $(8 \%)$, reflecting the tendency in recent years not to use older tetracyclines for the treatment of CARTIs. All isolates are susceptible to fluoroquinolones. Fluoroquinolones still remain among the antimicrobial agents that are most powerful against $H$. influenzae in vitro and are also highly effective as treatment of respiratory tract infections [37], although resistance has been recognized [38], and therapeutic failures in patients with community-acquired pneumonia have been associated with levofloxacin resistance in $H$. influenzae [39]. Overall, $47.8 \%$ of the $M$. catarrhalis isolates were $\beta$ lactamase positive. The $\beta$-lactamases which confer resistance to ampicillin have been characterized as BRO-1 and BRO-2 for $M$. catarrhalis and are inhibited by clavulanic acid and the combination of amoxicillin + clavulanic acid has been shown to be highly active against these species [40]. Since the first report of $\beta$-lactamase-producing M. catarrhalis in 1977 [41], the percentage of these strains has increased worldwide over the years, exceeding $90 \%$ in some countries $[8,31,32]$. Apart from macrolides and cotrimoxazole all antimicrobials had good in vitro activity against this organism.

In conclusion, there is a decreasing trend in the prevalence of resistance of the three most common pathogens involved in CARTIs. However, continuous surveillance at local and national levels remains important to detect any further changes in pathogens and monitor any changes in antimicrobial susceptibility among the major respiratory pathogens responsible for CARTIs.

\section{Conflict of Interests}

The authors declare that there is no conflict of interests regarding the publication of this paper.

\section{References}

[1] C. W. Stratton, "An overview of community-acquired respiratory tract infections," Advanced Studies in Pharmacy, vol. 2, no. 6, pp. 212-218, 2005.

[2] T. M. File Jr., J. Garau, F. Blasi et al., "Guidelines for empiric antimicrobial prescribing in community-acquired pneumonia," Chest, vol. 125, no. 5, pp. 1888-1901, 2004.

[3] K. P. Klugman, D. E. Low, J. Metlay, J.-C. Pechere, and K. Weiss, "Community-acquired pneumonia: new management strategies for evolving pathogens and antimicrobial susceptibilities," 
International Journal of Antimicrobial Agents, vol. 24, no. 5, pp. 411-422, 2004.

[4] C. Alpuche, J. Garau, and V. Lim, "Global and local variations in antimicrobial susceptibilities and resistance development in the major respiratory pathogens," International Journal of Antimicrobial Agents, vol. 30, no. 2, pp. 135-138, 2007.

[5] J.-H. Song and D. R. Chung, "Respiratory infections due to drug-resistant bacteria," Infectious Disease Clinics of North America, vol. 24, no. 3, pp. 639-653, 2010.

[6] J. Aspa, O. Rajas, and F. R. de Castro, "Pneumococcal antimicrobial resistance: therapeutic strategy and management in community-acquired pneumonia," Expert Opinion on Pharmacotherapy, vol. 9, no. 2, pp. 229-241, 2008.

[7] S. Tristram, M. R. Jacobs, and P. C. Appelbaum, "Antimicrobial resistance in Haemophilus influenzae," Clinical Microbiology Reviews, vol. 20, no. 2, pp. 368-389, 2007.

[8] T. F. Murphy and G. I. Parameswaran, "Moraxella catarrhalis, a human respiratory tract pathogen," Clinical Infectious Diseases, vol. 49, no. 1, pp. 124-131, 2009.

[9] Clinical and Laboratory Standards Institute (CLSI), "Performance standards for antimicrobial susceptibility testing; Twenty-second informational supplement," M100-S22, CLSI, Wayne, Pa, USA, 2012.

[10] Clinical and Laboratory Standards Institute (CLSI), Dilution and Disk Susceptibility Testing of Infrequently Isolated or Fastidious Bacteria, Approved guideline M45-A2. CLSI, Wayne, Pa, USA, 2nd edition, 2012.

[11] S. Kanavaki, S. Karabela, E. Marinis, and N. J. Legakis, "Antibiotic resistance of clinical isolates of Streptococcus pneumoniae in Greece," Journal of Clinical Microbiology, vol. 32, no. 12, pp. 3056-3058, 1994.

[12] S. Kanavaki, E. Mantadakis, S. Karabela et al., "Antimicrobial resistance of Streptococcus pneumoniae isolates in Athens, Greece," European Journal of Clinical Microbiology and Infectious Diseases, vol. 24, no. 10, pp. 693-696, 2005.

[13] L. R. Peterson, "Penicillins for treatment of pneumococcal pneumonia: does in vitro resistance really matter?" Clinical Infectious Diseases, vol. 42, no. 2, pp. 224-233, 2006.

[14] S. Maraki, E. Mantadakis, and G. Samonis, "Serotype distribution and antimicrobial resistance of adult Streptococcus pneumoniae clinical isolates over the period 2001-2008 in Crete, Greece," Chemotherapy, vol. 56, no. 4, pp. 325-332, 2010.

[15] S. Riedel, S. E. Beekmann, K. P. Heilmann et al., "Antimicrobial use in Europe and antimicrobial resistance in Streptococcus pneumoniae," European Journal of Clinical Microbiology and Infectious Diseases, vol. 26, no. 7, pp. 485-490, 2007.

[16] L. A. Mandell, R. G. Wunderink, A. Anzueto et al., "Infectious Diseases Society of America/American Thoracic Society Consensus Guidelines on the management of communityacquired pneumonia in adults," Clinical Infectious Diseases, vol. 44, supplement 2, pp. S27-S72, 2007.

[17] G. Poulakou, I. Katsarolis, I. Matthaiopoulou et al., "Nationwide surveillance of Streptococcus pneumoniae in Greece: patterns of resistance and serotype epidemiology," International Journal of Antimicrobial Agents, vol. 30, no. 1, pp. 87-92, 2007.

[18] A. Marchese and G. C. Schito, "Resistance patterns of lower respiratory tract pathogens in Europe," International Journal of Antimicrobial Agents, vol. 16, supplement 1, pp. S25-S29, 2000.
[19] D. J. Farrell, I. Morrissey, S. Bakker, and D. Felmingham, "Molecular characterization of macrolide resistance mechanisms among Streptococcus pneumoniae and Streptococcus pyogenes isolated from the PROTEKT 1999-2000 study," Journal of Antimicrobial Chemotherapy, vol. 50, supplement 1, pp. 39-47, 2002.

[20] M. Bergman, S. Huikko, P. Huovinen, P. Paakkari, H. Seppälä, and The Finnish Study Group for Antimicrobial resistance (FiRe Network), "Macrolide and azithromycin use are linked to increased macrolide resistance in Streptococcus pneumoniae," Antimicrobial Agents and Chemotherapy, vol. 50, no. 11, pp. 3646-3650, 2006.

[21] F. Baquero, G. Baquero-Artigao, R. Cantón, and C. García-Rey, "Antibiotic consumption and resistance selection Streptococcus pneumoniae," Journal of Antimicrobial Chemotherapy, vol. 50, supplement 2, pp. 27-37, 2002.

[22] C. García-Rey, L. Aguilar, F. Baquero, J. Casal, and R. DalRé, "Importance of local variations in antibiotic consumption and geographical differences of erythromycin and penicillin resistance in Streptococcus pneumoniae," Journal of Clinical Microbiology, vol. 40, no. 1, pp. 159-164, 2002.

[23] P. Kärpänoja, S. T. Nyberg, M. Bergman, The Finnish Study Group for Antimicrobial resistance (FiRe Network) et al., "Connection between trimethoprim-sulfamethoxazole and resistance in Streptococcus pneumoniae, Haemophilus influenzae, and Moraxella catarrhalis," Antimicrobial Agents and Chemotherapy, vol. 52, no. 7, pp. 2480-2485, 2008.

[24] M. W. Pletz, M. van der Linden, H. von Baum, C. B. Duesberg, K. P. Klugman, and T. Welte, "Low prevalence of fluoroquinolone resistant strains and resistance precursor strains in Streptococcus pneumoniae from patients with communityacquired pneumonia despite high fluoroquinolone usage," International Journal of Medical Microbiology, vol. 301, no. 1, pp. 5357, 2011.

[25] C. García-Rey, J. E. Martín-Herrero, and F. Baquero, "Antibiotic consumption and generation of resistance in Streptococcus pneumoniae: the paradoxical impact of quinolones in a complex selective landscape," Clinical Microbiology and Infection, vol. 12, supplement 3, pp. 55-66, 2006.

[26] H. J. Adam, D. J. Hoban, A. S. Gin, and G. G. Zhanel, "Association between fluoroquinolone usage and a dramatic rise in ciprofloxacin-resistant Streptococcus pneumoniae in Canada, 1997-2006," International Journal of Antimicrobial Agents, vol. 34, no. 1, pp. 82-85, 2009.

[27] E. J. C. Goldstein and S. M. Garabedian-Ruffalo, "Widespread use of fluoroquinolones versus emerging resistance in pneumococci," Clinical Infectious Diseases, vol. 35, no. 12, pp. 1505-1511, 2002.

[28] J. P. Lynch III and G. G. Zhanel, "Streptococcus pneumoniae: does antimicrobial resistance matter?" Seminars in Respiratory and Critical Care Medicine, vol. 30, no. 2, pp. 210-238, 2009.

[29] J. M. Blondeau, X. Zhao, G. Hansen, and K. Drlica, "Mutant prevention concentrations of fluoroquinolones for clinical isolates of Streptococcus pneumoniae," Antimicrobial Agents and Chemotherapy, vol. 45, no. 2, pp. 433-438, 2001.

[30] D. P. Kofteridis, G. Notas, S. Maraki et al., "Antimicrobial susceptibilities of 930 Haemophilus influenzae clinical strains isolated from the island of Crete, Greece," Chemotherapy, vol. 54, no. 6, pp. 492-498, 2008.

[31] M. R. Jacobs, D. Felmingham, P. C. Appelbaum, and R. N. Grüneberg, “The Alexander Project 1998-2000: susceptibility of 
pathogens isolated from community-acquired respiratory tract infection to commonly used antimicrobial agents," Journal of Antimicrobial Chemotherapy, vol. 52, no. 2, pp. 229-246, 2003.

[32] D. Hoban and D. Felmingham, "The PROTEKT surveillance study: antimicrobial susceptibility of Haemophilus influenzae and Moraxella catarrhalis from community-acquired respiratory tract infections," Journal of Antimicrobial Chemotherapy, vol. 50, supplement 1, pp. 49-59, 2002.

[33] H. Seki, Y. Kasahara, K. Ohta et al., "Increasing prevalence of ampicillin-resistant, non-beta-lactamase-producing strains of Haemophilus influenzae in children in Japan," Chemotherapy, vol. 45, no. 1, pp. 15-21, 1999.

[34] A. Jain, P. Kumar, and S. K. Agarwal, "High nasopharyngeal carriage of $\beta$-lactamase-negative ampicillin-resistant Haemophilus influenzae in north Indian school-going children," Journal of Infection and Chemotherapy, vol. 14, no. 1, pp. 72-74, 2008.

[35] M. R. Jacobs, "Increasing antibiotic resistance among otitis media pathogens and their susceptibility to oral agents based on pharmacodynamic parameters," Pediatric Infectious Disease Journal, vol. 19, 5, supplement, pp. S47-S56, 2000.

[36] A. Senok, M. Al-Zarouni, J. Al-Najjar, A. Nublusi, and D. Panigrahi, "Antimicrobial resistance among Streptococcus pneumoniae and Haemophilus influenzae isolates in the United Arab Emirates: 2004-2006," Journal of Infection in Developing Countries, vol. 1, no. 3, pp. 296-302, 2007.

[37] J. A. García-Rodríguez, F. Baquero, J. García de Lomas, and L. Aguilar, "Antimicrobial susceptibility of 1422 Haemophilus influenzae isolates from respiratory tract infections in Spain. Results of a 1 year (1996-97) multicenter surveillance study," Infection, vol. 27, no. 4-5, pp. 265-267, 1999.

[38] S. L. Barriere and J. A. Hindler, "Ciprofloxacin-resistant Haemophilus influenzae infection in a patient with chronic lung disease," Annals of Pharmacotherapy, vol. 27, no. 3, pp. 309-310, 1993.

[39] T. Bastida, M. Pérez-Vázquez, J. Campos et al., "Levofloxacin treatment failure in Haemophilus influenzae pneumonia," Emerging Infectious Diseases, vol. 9, no. 11, pp. 1475-1478, 2003.

[40] A. A. Kadry, S. I. Fouda, N. A. Elkhizzi, and A. M. Shibl, "Correlation between susceptibility and BRO type enzyme of Moraxella catarrhalis strains," International Journal of Antimicrobial Agents, vol. 22, no. 5, pp. 532-536, 2003.

[41] B. E. Malmvall, J. E. Brorsson, and J. Johnsson, "In vitro sensitivity to penicillin $\mathrm{V}$ and $\beta$ lactamase production of Branhamella catarrhalis," Journal of Antimicrobial Chemotherapy, vol. 3, no. 4, pp. 374-375, 1977. 


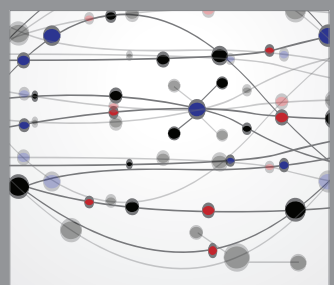

The Scientific World Journal
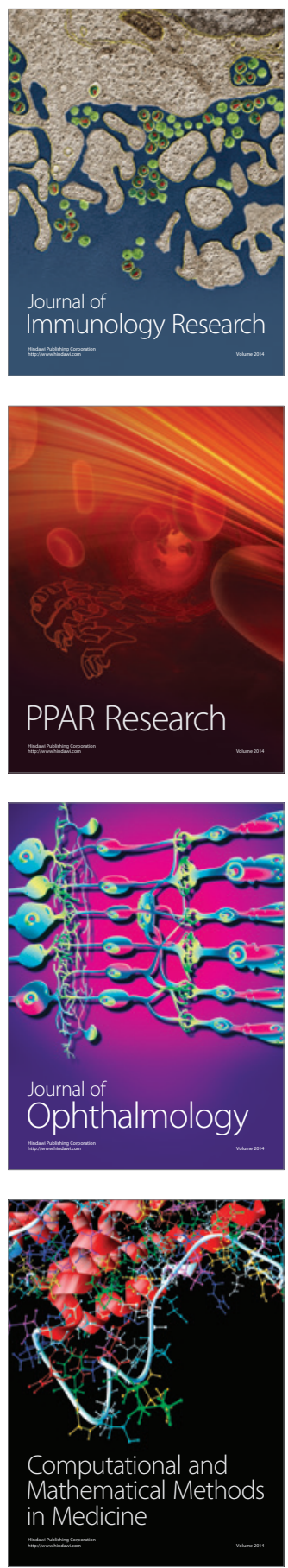

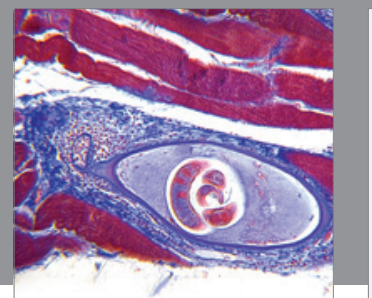

Gastroenterology

Research and Practice
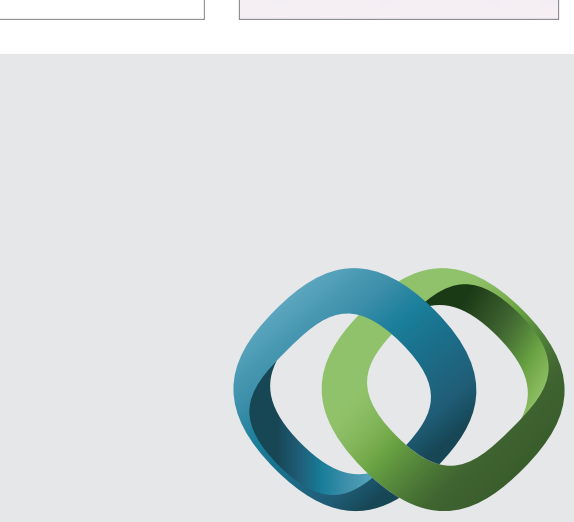

\section{Hindawi}

Submit your manuscripts at

http://www.hindawi.com
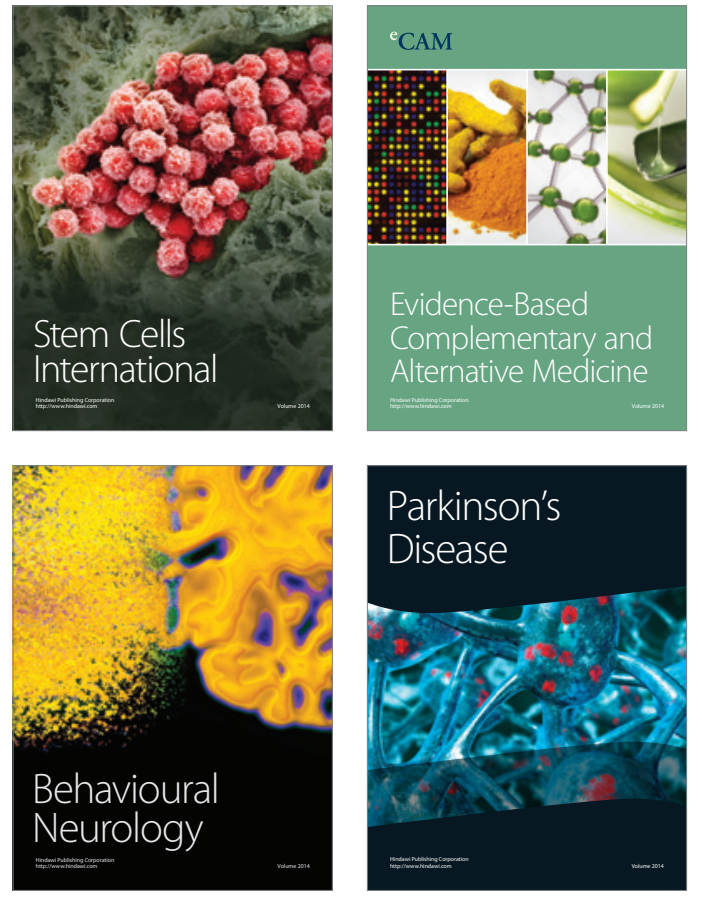
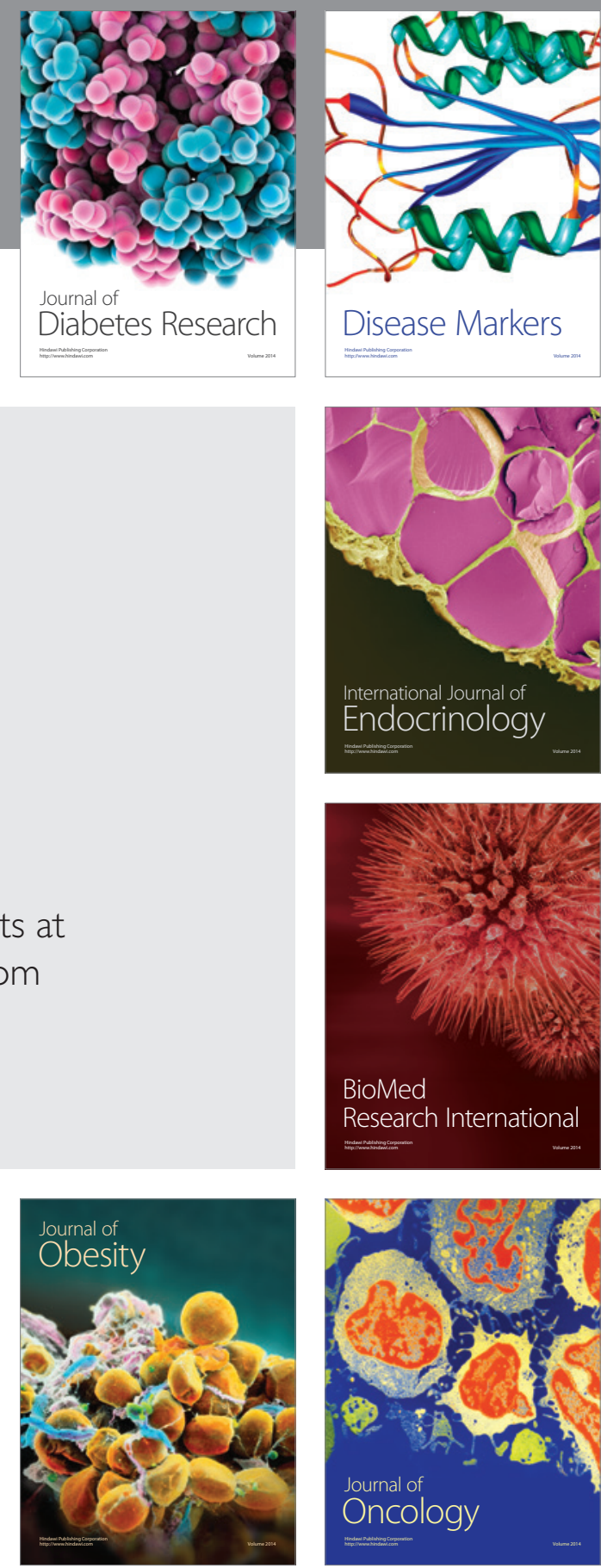

Disease Markers
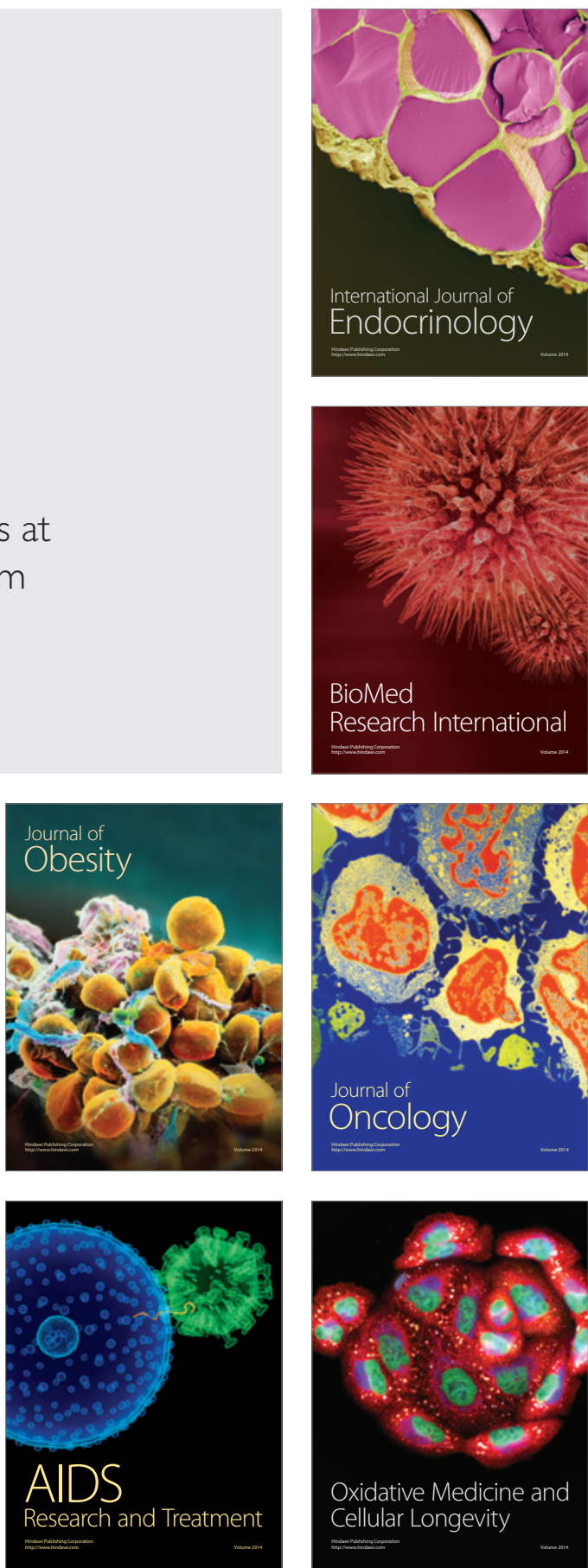